\title{
AN AIRBORNE STEREOSCOPIC MISSION TO EXPLORE THE 1998 AND 1999 LEONID METEOR STORMS
}

\author{
P. JENNISKENS, S. BUTOW \\ The SETI Institute at NASA/Ames Research Center \\ Mail Stop 239-4, Moffett Field, CA 94035-1000
}

The possibility of a storm of Leonid meteors in November 1998 and 1999 offers exciting opportunities for using unusual meteor observing techniques, because of high flux and because the Leonids are unique probes for the study of the parent comet 55P/Tempel-Tuttle and the Earth's upper atmosphere.

A drawback is the cloudy weather in mid November on the Northern hemisphere, which hampered all but radar observations during the previous storm in 1966.

For November 1998 and 1999, we have proposed to bring meteor astronomers, atmospheric physicists, and instrument scientists together on a multi-instrument multi-aircraft campaign. This will allow us to be above the clouds and low altitude haze, apply mid-infrared spectroscopy and imaging, and observe the same meteors by a wide range of techniques.

At present, ten experiments have been proposed that address fundamental questions in meteor physics (mass-luminosity relationship of fast meteors, chemistry and physics in the meteor trail and train), astronomy (meteoroid orbits, meteoroid flux, grain ejection velocities from the parent comet, dust composition, dust morphology), and atmospheric physics (deposition of neutral atoms, vertical transport, ambient temperatures and wind patterns in the mesosphere).

These issues will be addressed by optical imaging using photographic and video techniques for astrometry (P. Jenniskens, M. de Lignie, B. Hawkes), simultaneous ground-based radar (J. Jones, P. Brown), neutral atom detection (G. Swenson), lidar (C. Gardner), optical spectroscopy using photographic and video techniques ( $\mathrm{H}$. Betlem, J. Borovicka), mid-IR spectroscopy and imaging (R. Russell, J. Turk) and imaging of the sky in the direction of the true radiant of the Leonid stream (B. Gustafson).

The first validation flights were executed on August 11 and 12, 1997, with the support of the US Air Force at Edwards Air Force Base, CA. 